\title{
Fixed combination of lercanidipine and enalapril in the management of hypertension: focus on patient preference and adherence
}

This article was published in the following Dove Press journal:

Patient Preference and Adherence

16 June 2012

Number of times this article has been viewed

\author{
Claudio Borghi \\ Francesca Santi \\ Internal Medicine, Aging and Kidney \\ Disease Department, University \\ of Bologna, Italy
}

\begin{abstract}
Hypertension is one of the most important and widespread risk factors for the development of cardiovascular disease. Once, combination therapy was traditionally reserved as a third-line or fourth-line approach in the management of hypertension. However, several major intervention trials in high-risk patient populations have shown that an average of 2-4 antihypertensive agents are required to achieve effective blood pressure control. Combination treatment should be considered as a first choice in patients at high cardiovascular risk and in individuals for whom blood pressure is markedly above the hypertension threshold (eg, more than $20 \mathrm{mmHg}$ systolic or $10 \mathrm{mmHg}$ diastolic), or when milder degrees of blood pressure elevation are associated with multiple risk factors, subclinical organ damage, diabetes, renal failure, or associated cardiovascular disease. A number of clinical trials have demonstrated that a fixed combination of lercanidipine and enalapril has better efficacy and tolerability than monotherapy with either agents. The fixed-dose formulation of lercanidipine-enalapril was well tolerated in all clinical trials, with an adverse event rate similar to that of the component drugs as monotherapy. The advantages of combination therapy include improved adherence to therapy and minimization of blood pressure variability. In addition, combining two antihypertensive agents with different mechanisms of action may provide greater protection against major cardiovascular events and the development of end-organ damage.
\end{abstract}

Keywords: hypertension, treatment, fixed-dose combination, lercanidipine, enalapril

\section{Introduction}

Hypertension is one of the most important and widespread cardiovascular risk factors responsible for the development of cerebrovascular disorders, heart disease, and renal failure. Combination therapy was traditionally reserved as a third-line or fourth-line approach in the management of hypertension. ${ }^{1}$ However, several major intervention trials in high-risk patient populations have shown that an average of 2-4 antihypertensive agents are required to achieve effective blood pressure control. ${ }^{2-8}$ The European Society of Hypertension guidelines ${ }^{9}$ comment on the possibility of starting antihypertensive treatment with a single drug at low dose or with combination therapy. In fact, the socalled "responder rate" (systolic and diastolic blood pressure reduction $\geq 20 \mathrm{mmHg}$ and $10 \mathrm{mmHg}$, respectively) to any agent in monotherapy is approximately $50 \%,{ }^{10}$ and the ability of any agent used alone to achieve target blood pressure values $(<140 / 90 \mathrm{mmHg})$ does not exceed $20 \%-30 \%$ in the overall hypertensive population, except in subjects with grade 1 hypertension. ${ }^{11,12}$

In most trials, a combination of two or more drugs has been the most widely used treatment regimen to reduce blood pressure effectively. Combination therapy
Internal Medicine, Aging and Kidney

Disease Department, University

of Bologna, Via Albertoni I5,

Bologna 40138 , Italy

Fax +39051390646

Tel +390516362212

Email francesca.snt.santi@gmail.com 
has been found to be more effective in high-risk patients and diabetics, and whenever lower blood pressure targets are needed. ${ }^{13}$

An obvious disadvantage of initiating treatment with two drugs is the potential exposure of some patients to an unnecessary agent. However, the advantages seem to be overwhelming. In fact, by using a combination, both drugs can be given in lower dosage, thus minimizing the risk of side effects compared with full-dose monotherapy. Furthermore, fixed low-dose combinations are available, allowing two agents to be administered in a single tablet, both simplifying and optimizing treatment and compliance. The advantages of combination therapy are well documented, with increased antihypertensive efficacy as a result of the simultaneous inhibition of different mechanisms of action, with a lesser incidence of adverse events, because of the possible compensatory responses and the lower doses used. ${ }^{14}$

Starting treatment with a two-drug combination therapy may allow blood pressure control to be achieved in a shorter time. This may be of critical importance in high-risk patients, because the VALUE (Valsartan Antihypertensive Long-term Use Evaluation) trial demonstrated that in the first 6 months of treatment, a greater blood pressure reduction $(23.8 / 22.2 \mathrm{mmHg})$ obtained in amlodipine-treated versus valsartan-treated patients was accompanied by a difference in cardiovascular event rate in favor of the more effectively treated group. ${ }^{4,9}$

Combination treatment should be considered as the first choice in patients at high cardiovascular risk and in individuals for whom blood pressure is markedly above the hypertension threshold (eg, more than $20 \mathrm{mmHg}$ systolic or $10 \mathrm{mmHg}$ diastolic), or when milder degrees of blood pressure elevation are associated with multiple risk factors, subclinical organ damage, diabetes, renal failure, or associated cardiovascular disease. ${ }^{9}$

In all these conditions, the need to obtain a larger blood pressure reduction could not be satisfied by monotherapy alone, and often more than two drugs are needed. Guidelines recommend various two-drug combinations of different classes of antihypertensive agents based on data derived from controlled interventional trials, but 3-4 drugs may be required depending on the patient's risk profile.

In clinical practice, numerous fixed-dose antihypertensive combination regimens are widely available, ie, a betablocker + hydrochlorothiazide, an angiotensin-converting enzyme inhibitor + hydrochlorothiazide, an angiotensin receptor blocker + hydrochlorothiazide, and a calcium channel blocker + angiotensin-converting enzyme inhibitor. ${ }^{15}$
Many studies have shown that newer antihypertensive agents, such as calcium channel blockers, angiotensin receptor blockers, and angiotensin-converting enzyme inhibitors, provide additional benefits by reducing the incidence of cardiovascular events in patients with hypertension. ${ }^{4,16-18}$ This review focuses on the fixed association of lercanidipineenalapril, pointing out the potential advantages of such a combination.

\section{Lercanidipine}

Calcium channel blockers are extensively used in clinical practice, and several randomized clinical trials have shown that calcium channel blockers are potent antihypertensive drugs with good tolerability both in the general hypertensive population and in a wide range of patients, alone or in combination. ${ }^{14,19}$

Lercanidipine is a third-generation dihydropyridine calcium channel blocker which inhibits calcium entry through L-type calcium channels in smooth muscle cells of the cardiovascular system, leading to peripheral vasodilatation ${ }^{20-22}$ and so exerting its antihypertensive effect. It is a highly lipophilic drug and has a slower onset and longer duration of action than other dihydropyridines. ${ }^{23}$ Furthermore, the drug is highly vasoselective because of the high proportion of L-type calcium channels in arteries and has shown less in vitro and in vivo negative inotropic activity than some other dihydropyridines. ${ }^{20}$ Lercanidipine is a well tolerated drug with a low adverse event rate due to its long-lasting and vasoselective calcium entry blocking activity, and does not cause sympathetic activation and reflex tachycardia. ${ }^{24}$ As a result, the overall adverse event rate is lower than that observed with other dihydropyridines. ${ }^{15}$ The efficacy of lercanidipine has been evaluated in both noncomparative and comparative studies with other calcium channel blockers and different antihypertensive drugs, showing comparable effects in all cases. ${ }^{25-30}$

Some studies have suggested that lercanidipine may have antiatherogenic effects beyond blood pressure reduction. ${ }^{21,31-33}$ Another reported benefit with lercanidipine is its renoprotective effect, which is related to its ability to induce both afferent and efferent arteriolar vasodilatation. ${ }^{34,35}$ Lercanidipine was also superior to ramipril in reducing albumin excretion in diabetic patients with microalbuminuria. ${ }^{35}$

In diabetic patients with hypertension, treatment with lercanidipine was able to decrease the glycosylated hemoglobin level significantly, without negatively affecting glucose homeostasis, to enhance glucose tolerance, and to reduce fasting blood glucose, with either neutral or favorable effects on 
the lipid profile. ${ }^{22,36}$ Moreover, in diabetics with renal failure, lercanidipine had a good tolerability profile and a neutral effect on plasma lipids, with no impairment of renal function. ${ }^{37}$

In hypertensive patients with metabolic syndrome, lercanidipine appeared to have a better tolerability profile and was associated with fewer vasodilatation-related adverse effects than other dihydropyridine calcium channel blockers. ${ }^{15}$ Lercanidipine also reduces the signs and symptoms of ischemia, and improves heart function in patients with angina. ${ }^{38}$

\section{Enalapril}

The renin-angiotensin-aldosterone system plays a key role in regulating the homeostasis of fluids, electrolytes, and systemic vascular resistance. Overactivation of this system, especially through excessive production of its effector peptide, angiotensin II, has been related to the genesis and development of cardiovascular disease. Angiotensin-converting enzyme inhibitors are able to diminish plasma levels of angiotensin II by blocking the last step of its activation. This results in reduced vascular resistance, leading to a decrease in blood pressure values. ${ }^{14,39}$

Enalapril, one the most commonly prescribed angiotensinconverting enzyme inhibitors in clinical practice in a number of European countries, is an orally administered prodrug that is hydrolyzed to the active metabolite, enalaprilat, which decreases plasma levels of angiotensin II by inhibiting the last step of its activation. The reduction of angiotensin II leads to peripheral vasodilatation and reduced vascular resistance, decreasing blood pressure values. ${ }^{21,39,40}$ Enalapril is a dosedependent antihypertensive drug, with its maximum effect occurring 6-8 hours after administration and a total duration of effect of 24-36 hours. ${ }^{40}$ Enalapril has been shown to be an effective antihypertensive agent, with positive effects on cardiovascular risk factors, prevention of decline in renal function and other organ damage, ${ }^{41-44}$ like progression of intima media thickness in the carotid artery, an independent risk factor for cardiovascular and cerebrovascular disease. ${ }^{43}$ The cardiovascular protection associated with enalapril may be caused by potentiation of the effects of bradykinin. ${ }^{45}$ Further, in a randomized, double-blind, six-year trial in patients with diabetes and normoalbuminuria at baseline, enalapril reduced the development of microalbuminuria. ${ }^{42}$

\section{Combination of enalapril- lercanidipine}

Because calcium channel blockers do not share the mode of action of renin-angiotensin-aldosterone system inhibitors, a combination of these agents should provide synergistic and complementary effects. Indeed, in patients with newly diagnosed stage 1 or 2 hypertension and in patients with inadequate blood pressure control after conventional low-dose monotherapy, combination therapy with a calcium channel blocker and an angiotensin-converting enzyme inhibitor may be particularly effective. ${ }^{46,47}$

Calcium channel blockers are potent vasodilators that induce reflex activation of the sympathetic system and the renin-angiotensin-aldosterone system. As a result, the use of an angiotensin-converting enzyme inhibitor may buffer this excessive activation. Moreover, since calcium channel blockers promote a negative sodium balance and an increase in angiotensin II levels, this may reinforce the antihypertensive effect of angiotensin-converting enzyme inhibition. ${ }^{48}$ On the other hand, the concomitance of both treatments may reduce the incidence of adverse events, in particular peripheral edema, due to an increase in intracapillary pressure as a consequence of selective diminution of precapillary arteriolar tone during blockade of calcium entry. Angiotensin-converting enzyme inhibitors reduce the lower extremity edema caused by calcium channel blockers, likely because of their ability to dilate both the arterial vascular bed and the venous capacitance vessels. ${ }^{49}$

In the SELECT (Systolic Evaluation of Lotrel Efficacy and Comparative Therapies) study, calcium channel blocker and angiotensin-converting enzyme inhibitor combination therapy with amlodipine and benazepril, respectively, was significantly more effective in reducing systolic blood pressure and pulse pressure in patients with severe systolic hypertension than either agent used alone. ${ }^{50}$ The combination of manidipine and delapril was also more effective in reducing blood pressure than either drug used alone, achieving blood pressure control in $73 \%$ of treated patients. ${ }^{51}$

Data from the trials show that calcium channel blocker therapy plus additional addon treatment is able to not only lower blood pressure, but also to improve patient outcomes. The HOT (Hypertension Optimal Treatment) trial showed that intensive lowering of blood pressure with calcium channel blocker-based therapy led to a low rate of cardiovascular events. ${ }^{52}$ In the Syst-Eur (Systolic Hypertension in Europe) and Syst-China (Systolic Hypertension in China) studies, nitrendipine plus a diuretic and an angiotensinconverting enzyme inhibitor reduced the rate of cardiovascular complications in elderly patients with isolated systolic hypertension..$^{53,54}$

Data from the ASCOT study showed that treatment with amlodipine plus perindopril (an angiotensin-converting 
enzyme inhibitor, added as required) versus atenolol (a beta-blocker) plus bendroflumethiazide (a diuretic, added as required) was more effective in reducing the risks of nonfatal myocardial infarction or fatal coronary heart disease, fatal and nonfatal stroke, total cardiovascular events and procedures, all-cause mortality, and diabetes in patients with hypertension and at least three other cardiovascular risk factors. ${ }^{55}$ The ACCOMPLISH (Avoiding Cardiovascular Events through Combination Therapy in Patients Living with Systolic Hypertension) trial compared the effectiveness of a maximally titrated, fixed-dose combination of benazepril (an angiotensin-converting enzyme inhibitor) and amlodipine (a dihydropyridine calcium channel blocker) with the combination of benazepril and hydrochlorothiazide in reducing cardiovascular morbidity and mortality. The trial was stopped early because of a $20 \%$ reduction in cardiovascular risk recorded in the benazepril plus amlodipine group, so demonstrating that combination treatment with benazepril plus amlodipine reduces progression of chronic kidney disease and cardiovascular or all-cause mortality in high-risk hypertensive patients. Differences in blood pressure control throughout the study could not account for these findings. ${ }^{56}$

A number of clinical trials have demonstrated that the fixed combination of lercanidipine and enalapril has better efficacy and tolerability than monotherapy with either agent $^{57-60}$ (Table 1). In a trial performed in hypertensive patients nonresponsive to lercanidipine, after 12 weeks of treatment with fixed-combination lercanidipine-enalapril, a significantly greater proportion of patients had normalized blood pressure compared with patients treated with lercanidipine as monotherapy $(22 \%$ versus $12 \%, P=0.012) .{ }^{59}$ Similarly, in another study carried out in hypertensive patients nonresponsive to enalapril, after 12 weeks of treatment with this fixed combination, there was a trend towards better blood pressure control in the population treated with the fixed combination compared with enalapril as monotherapy $(24 \%$ versus $17 \%){ }^{58}$

A randomized, double-blind, placebo-controlled study showed that reduction in blood pressure was greater in patients who received the lercanidipine-enalapril combination than in those assigned to receive either component as monotherapy. ${ }^{6}$ In fact, all active treatments significantly reduced mean 24-hour blood pressure and systolic blood pressure in the office compared with placebo, but the lercanidipine-enalapril combination was significantly more effective than the active components as monotherapy. Moreover, a higher proportion of patients treated with the lercanidipine-enalapril combination achieved their target blood pressure compared with those treated with lercanidipine or enalapril alone (45\% versus $18 \%$ versus $19 \%$, respectively). In addition, lercanidipine was noninferior to hydrochlorothiazide as addon therapy in diabetic patients with hypertension who had not responded to enalapril alone. ${ }^{57}$

The fixed-dose formulation of lercanidipine-enalapril was well tolerated in all published clinical trials, with an adverse effect rate similar to that of the component drugs as monotherapy. ${ }^{57-60}$ Adverse effects were generally transient and of mild severity, and there were no reports of peripheral edema. ${ }^{57-60}$ Although infrequent, the most prevalent adverse events related to the use of the lercanidipine-enalapril combination were cough, dizziness, and vertigo. Interestingly, no clinically significant differences in heart rate were observed between the treatment groups, and 24-hour heart rate remained stable on all treatments. ${ }^{60}$ The absence of negative effects of the combination on lipid and glucose metabolism appears to be an added advantage in the treatment of hypertensive population. ${ }^{39}$

Table I Effect of fixed association of enalapril-lercanidipine on blood pressure control

\begin{tabular}{|c|c|c|c|c|}
\hline Reference & Patients, $\mathrm{n}$ & Duration & Intervention & Outcome \\
\hline \multirow[t]{2}{*}{ Agrawal et al ${ }^{57}$} & 174 & 20 weeks & Lercanidipine + enalapril 20 mg versus & $\mathrm{SBP}-9.6$ versus $-6.0 \mathrm{mmHg}$ \\
\hline & & & hydrochlorothiazide + enalapril 20 mg & DBP -9.3 versus $-7.4 \mathrm{mmHg}$ \\
\hline \multirow[t]{2}{*}{ Recordati SpA ${ }^{58}$} & 338 & 12 weeks & Enalapril $20 \mathrm{mg}$ versus lercanidipine & $\mathrm{SBP}-6.7$ versus $-9.8 \mathrm{mmHg}$ \\
\hline & & & $10 \mathrm{mg}+$ enalapril $20 \mathrm{mg}$ & DBP -7.5 versus $-9.2 \mathrm{mmHg}$ \\
\hline \multirow[t]{2}{*}{ Recordati SpA ${ }^{59}$} & 337 & 12 weeks & Lercanidipine $10 \mathrm{mg}$ versus lercanidipine & $\mathrm{SBP}-2.3$ versus $-7.7 \mathrm{mmHg}$ \\
\hline & & & $10 \mathrm{mg}+$ enalapril $10 \mathrm{mg}$ & $\mathrm{DBP}-4.3$ versus $-7.1 \mathrm{mmHg}$ \\
\hline \multirow[t]{2}{*}{ Puig et $\mathrm{al}^{60}$} & 75 & 4 months & Lercanidipine $10 \mathrm{mg}$ versus enalapril $20 \mathrm{mg}$ & SBP -5 versus -5.9 versus \\
\hline & & & alone or in combination versus placebo & $16.9 \mathrm{mmHg}$, versus placebo \\
\hline \multirow[t]{2}{*}{ Rump ${ }^{61}$} & 8440 & 3 months & Enalapril $10 \mathrm{mg}+$ lercanidipine $10 \mathrm{mg}$ or & $\mathrm{SBP}-28.4 \mathrm{mmHg}$ \\
\hline & & & enalapril 10 mg + lercanidipine 20 mg & $\mathrm{DBP}-13.5 \mathrm{mmHg}$ \\
\hline \multirow[t]{2}{*}{ Gil Guillén et al ${ }^{65}$} & 1562 & 6 months & Lercanidipine + enalapril $20 \mathrm{mg}$ alone & $\mathrm{SBP}-25.4 \mathrm{mmHg}$ \\
\hline & & & or in combination & $\mathrm{DBP}-13.6 \mathrm{mmHg}$ \\
\hline
\end{tabular}

Abbreviations: SBP, systolic blood pressure; DBP, diastolic blood pressure. 
In an observational study which examined more than 8000 patients, physician (general practitioners and specialist in internal medicine) subjective assessment of the lercanidipine-enalapril combination was positive, the efficacy of the fixed combination was assessed by $94 \%$ as "very good" to "good". The physicians also assessed tolerability in $97 \%$ of the patients as "very good" or "good", and assessed compliance as "very good" or "good" in 97\% of patients. ${ }^{61}$

\section{Conclusion}

The main advantages of combination therapy include improved adherence to therapy ${ }^{62}$ and minimization of blood pressure variability. In addition, combining two antihypertensive agents with different mechanisms of action may provide greater protection against major cardiovascular events and end-organ damage. ${ }^{9}$ Combinations of two drugs in a single tablet, usually at low doses, (but sometimes at both lower and higher doses), are now widely available. Although the fixed doses of the components in the combination limits the flexibility of upward and downward treatment strategies, fixed combinations reduce the number of tablets to be taken by the patient, and this has some advantage for compliance with treatment. ${ }^{62,63}$

Fixed-dose combinations can substitute extemporaneous combinations that have successfully controlled blood pressure, but, when used at low doses, they can also be considered for first-step treatment. ${ }^{9}$ Guidelines recommend various twodrug combinations of different classes of antihypertensive agents based on data derived from controlled interventional trials, but advise that 3-4 drugs may be required, depending on the patient's risk profile.

In contrast, many studies have shown that newer antihypertensive agents, including calcium channel blockers, angiotensin receptor blockers, and angiotensin-converting enzyme inhibitors, provide additional benefits by reducing the incidence of cardiovascular events in patients with hypertension. ${ }^{4,16-18}$ In addition, cases of new-onset diabetes are less common with newer antihypertensive agents than with older therapies, such as diuretics and beta-blockers. ${ }^{64}$ Whether this is due to the deleterious effect of the older agents on glucose metabolism or to a positive effect of the newer agents remains to be clarified. ${ }^{19} \mathrm{~A}$ fixed combination of lercanidipine and enalapril has been shown to be effective in controlling calcium channel blocker levels and data about tolerability and patient compliance, indicating that this combination is a suitable, effective, and safe treatment for hypertension.

\section{Disclosure}

The authors report no conflicts of interest in this work.

\section{References}

1. Chobanian AV, Bakris GL, Black HR, et al. The Seventh Report of the Joint National Committee on Prevention, Detection, Evaluation, and Treatment of High Blood Pressure: the JNC 7 report. JAMA. 2003;289:2560-2572.

2. UK Prospective Diabetes Study Group. Tight blood pressure control and risk of macrovascular and microvascular complications in type 2 diabetes: UKPDS 38. Br Med J. 1998;317:703-713.

3. Hansson L, Zanchetti A, Carruthers SG, et al. Effects of intensive bloodpressure lowering and low-dose aspirin in patients with hypertension: principal results of the Hypertension Optimal Treatment (HOT) randomised trial. HOT Study Group. Lancet. 1998;351:1755-1762.

4. Julius S, Kjeldsen SE, Weber M, et al. Outcomes in hypertensive patients at high cardiovascular risk treated with regimens based on valsartan or amlodipine: the VALUE randomised trial. Lancet. 2004;363:2022-2031.

5. Lewis EJ, Hunsicker LG, Clarke WR, et al. Renoprotective effect of the angiotensin-receptor antagonist irbesartan in patients with nephropathy due to type 2 diabetes. $N$ Engl J Med. 2001;345:851-860.

6. Estacio RO, Schrier RW. Antihypertensive therapy in type 2 diabetes: implications of the appropriate blood pressure control in diabetes (ABCD) trial. Am J Cardiol. 1998;2:R1-R14.

7. Lazarus JM, Bourgoignie JJ, Buckalew VM, et al. Achievement and safety of a low blood pressure goal in chronic renal disease. The Modification of Diet in Renal Disease Study Group. Hypertension. 1997;29:641-650.

8. Agodoa LY, Appel L, Bakris GL, et al. Effect of ramipril vs amlodipine on renal outcomes in hypertensive nephrosclerosis: a randomized controlled trial. JAMA. 2001;285:2719-2728.

9. Mancia G, De Backer G, Dominiczak A, et al. The task force for the management of arterial hypertension of the European Society of Hypertension, The task force for the management of arterial hypertension of the European Society of Cardiology. 2007 Guidelines for the management of arterial hypertension: The Task Force for the Management of Arterial Hypertension of the European Society of Hypertension (ESH) and of the European Society of Cardiology (ESC). Eur Heart J. 2007;28:1462-1536.

10. Materson BJ, Reda DJ, Cushman WC. Department of Veterans Affairs single-drug therapy of hypertension study. Revised figures and new data. Department of Veterans Affairs Cooperative Study Group on Antihypertensive Agents. Am J Hypertens. 1995;8:189-192.

11. Morgan TO, Anderson AI, MacInnis RJ. ACE inhibitors, beta-blockers, calcium blockers, and diuretics for the control of systolic hypertension. Am J Hypertens. 2001;14:241-247.

12. Dickerson JE, Hingorani AD, Ashby MJ, Palmer CR, Brown MJ. Optimisation of antihypertensive treatment by crossover rotation of four major classes. Lancet. 1999;353:2008-2013.

13. Hansson L, Zanchetti A, Carruthers SG, et al. Effects of intensive blood pressure lowering and low-dose aspirin in patients with hypertension: principal results of the Hypertension Optimal Treatment (HOT) randomised trial. Lancet. 1998;351:1755-1762.

14. Barrios V, Escobar C, Echarri R. Fixed combinations in the management of hypertension: perspectives on lercanidipine - enalapril. Vasc Health Risk Manag. 2008;4:847-853.

15. Barrios V, Escobar C, de la Figuera M, et al. High doses of lercanidipine are better tolerated than other dihydropyridines in hypertensive patients with metabolic syndrome: results from the TOLERANCE study. Int $J$ Clin Pract. 2008;62:723-728.

16. Dahlof B, Devereux RB, Kjeldsen SE, et al. Cardiovascular morbidity and mortality in the Losartan Intervention For Endpoint reduction in hypertension study (LIFE): a randomised trial against atenolol. Lancet. 2002;359:995-1003. 
17. Yusuf S, Sleight P, Pogue J, Bosch J, Davies R, Dagenais G. Effects of an angiotensin-converting-enzyme inhibitor, ramipril, on cardiovascular events in high-risk patients. The Heart Outcomes Prevention Evaluation Study Investigators. N Engl J Med. 2000;342:145-153.

18. Brenner BM, Cooper ME, de Zeeuw D, et al. Effects of losartan on renal and cardiovascular outcomes in patients with type 2 diabetes and nephropathy. N Engl J Med. 2001;345:861-869.

19. Haller H. Effective management of hypertension with dihydropyridine calcium channel blocker-based combination therapy in patients at high cardiovascular risk. Int J Clin Pract. 2008;62:781-790.

20. McClellan KJ, Jarvis B. Lercanidipine: a review of its use in hypertension. Drugs. 2000;60:1123-1140.

21. Otero M. Manidipine-delapril combination in the management of hypertension. Vasc Health Risk Manag. 2007;3:255-263.

22. Bang LM, Chapman TM, Goa KL. Lercanidipine: a review of its efficacy in the management of hypertension. Drugs. 2003;63:2449-2472.

23. Meredith PA. Lercanidipine: a novel lipophilic dihydropyridine calcium antagonist with long duration of action and high vascular selectivity. Expert Opin Investig Drugs. 1999;8:1043-1062.

24. Epstein M. Lercanidipine: a novel dihydropyridine calcium-channel blocker. Heart Dis. 2001;3:398-407.

25. Barrios V, Navarro A, Esteras A, et al. Antihypertensive efficacy and tolerability of lercanidipine in daily clinical practice. The ELYPSE Study. Blood Press. 2002;11:95-100.

26. Barrios V, Escobar C, Navarro A, et al. Lercanidipine is an effective and well tolerated antihypertensive drug regardless the cardiovascular risk profile: The LAURA study. Int J Clin Pract. 2006;60:1364-1370.

27. James IG, Jones A, Davies P. A randomised, double-blind, doubledummy comparison of the efficacy and tolerability of lercanidipine tablets and losartan tablets in patients with mild to moderate essential hypertension. J Hum Hypertens. 2002;16:605-610.

28. Leonetti G, Magnani B, Pessina AC, et al. Tolerability of long-term treatment with lercanidipine versus amlodipine and lacidipine in elderly hypertensives. Am J Hypertens. 2002;5:932-940.

29. Cherubini A, Fabris F, Ferrari E, et al. Comparative effects of lercanidipine, lacidipine, and nifedipine gastrointestinal therapeutic system on blood pressure and heart rate in elderly hypertensive patients: the ELderly and LErcanidipine (ELLE) study. Arch Gerontol Geriatr. 2003;37:203-212.

30. Romito R, Pansini MI, Perticone F, et al. Comparative effect of lercanidipine, felodipine, and nifedipine GITS on blood pressure and heart rate in patients with mild to moderate arterial hypertension: the Lercanidipine in Adults (LEAD) Study. J Clin Hypertens. 2003;5:249-253.

31. Soma MR, Natali M, Donetti E, et al. Effect of lercanidipine and its (R)enantiomer on atherosclerotic lesions induced in hypercholesterolemic rabbits. Br J Pharmacol. 2003;125:1471-1476.

32. Rachmani R, Levi Z, Zadok BS, et al. Losartan and lercanidipine attenuate low-density lipoprotein oxidation in patients with hypertension and type 2 diabetes mellitus: a randomized, prospective crossover study. Clin Pharmacol Ther. 2002;72:302-307.

33. Canavesi $\mathrm{M}$, Baldini $\mathrm{N}$, Leonardi $\mathrm{A}$, et al. In vitro inhibitory effect of lercanidipine on cholesterol accumulation and matrix metalloproteinases secretion by macrophages. $J$ Cardiovasc Pharmacol. 2004; 44:416-422.

34. Robles NR, Ocon J, Gomez CF, et al. Lercanidipine in patients with chronic renal failure: the ZAFRA study. Ren Fail. 2005;27:73-80.

35. Dalla Vestra M, Pozza G, Mosca A, et al. Effect of lercanidipine compared with ramipril on albumin excretion rate in hypertensive type 2 diabetic patients with microalbuminuria: DIAL study (Diabete, Ipertensione, Albuminuria, Lercanidipina). Diabetes Nutr Metab. 2004; 17:259-266.

36. Viviani GL. Lercanidipine in type II diabetic patients with mild to moderate arterial hypertension. J Cardiovasc Pharmacol. 2002;40: 133-139.

37. Robles NR, Pastor L, Manjon M, et al. Lercanidipine in diabetic patients with renal failure. Nefrologia. 2004;24:338-343.
38. Acanfora D, Gheorghiade M, Trojano L, et al. A randomized, doubleblind comparison of lercanidipine 10 and $20 \mathrm{mg}$ in patients with stable effort angina: clinical evaluation of cardiac function by ambulatory ventricular scintigraphic monitoring. Am J Ther. 2004;11:423-432.

39. Hair PI, Scott LJ, Perry CM. Fixed-dose combination lercanidipine/ enalapril. Drugs. 2007;67:95-106.

40. Todd PA, Heel RC. Enalapril. A review of its pharmacodynamic and pharmacokinetic properties, and therapeutic use in hypertension and congestive heart failure. Drugs. 1986;31:198-248.

41. Todd PA, Goa KL. Enalapril. A reappraisal of its pharmacology and therapeutic use in hypertension. Drugs. 1992;43:346-381.

42. Ravid M, Brosh D, Levi Z, et al. Use of enalapril to attenuate decline in renal function in normotensive, normoalbuminuric patients with type 2 diabetes mellitus. A randomized, controlled trial. Ann Intern Med. 1998;128:982-988.

43. Hosomi N, Mizushige K, Ohyama H, et al. Angiotensin-converting enzyme inhibition with enalapril slows progressive intima-media thickening of the common carotid artery in patients with non-insulindependent diabetes mellitus. Stroke. 2001;32:1539-1545.

44. Rosei EA, Rizzoni D, Muiesan ML, et al. Effects of candesartan cilexetil and enalapril on inflammatory markers of atherosclerosis in hypertensive patients with non-insulin-dependent diabetes mellitus. J Hypertens. 2005;23:435-444.

45. Minai K, Matsumoto T, Horie H, et al. Bradykinin stimulates the release of tissue plasminogen activator in human coronary circulation: effects of angiotensin-converting enzyme inhibitors. J Am Coll Cardiol. 2001;37:1565-1570.

46. McInnes GT. Antihypertensive drugs in combination: additive or greater than additive? J Hum Hypertens. 2007;21:914-916.

47. Andreadis EA, Tsourous GI, Marakomichelakis GE, et al. High-dose monotherapy vs low-dose combination therapy of calcium channel blockers and angiotensin receptor blockers in mild to moderate hypertension. J Hum Hypertens. 2005;19:491-496.

48. Gojanovic B, Feihl F, Liaudet L, et al. Concomitant calcium entry blockade and inhibition of the renin-angiotensin system: a rational and effective means for treating hypertension. J Renin Angiotensin Aldosterone Syst. 2008;9:1-9.

49. Messerli FH. Vasodilatory edema: a common side effect of antihypertensive therapy. Curr Cardiol Rep. 2002;4:479-482.

50. Neutel JM, Smith DH, Weber MA, Schofield L, Purkayastha D, Gatlin M. Efficacy of combination therapy for systolic blood pressure in patients with severe systolic hypertension: the Systolic Evaluation of Lotrel Efficacy and Comparative Therapies (SELECT) study. J Clin Hypertens. 2005;7:641-646.

51. Mugellini A, Vaccarella A, Celentano A, Scanferla F, Zoppi A, Fogari R. Fixed combination of manidipine and delapril in the treatment of mild to moderate essential hypertension: evaluation by 24 -hour ambulatory blood pressure monitoring. Blood Press Suppl. 2005;1:6-13.

52. Amar J, Vaur L, Perret M, Bailleau C, Etienne S, Chamontin B. Hypertension in high-risk patients: beware of the underuse of effective combination therapy (results of the PRATIK study). J Hypertens. 2002;20:779-784.

53. Staessen JA, Fagard R, Thijs L, et al. Randomised double-blind comparison of placebo and active treatment for older patients with isolated systolic hypertension. The Systolic Hypertension in Europe (Syst-Eur) Trial Investigators. Lancet. 1997;350:757-764.

54. Liu L, Wang JG, Gong L, Liu G, Staessen JA. Comparison of active treatment and placebo in older Chinese patients with isolated systolic hypertension. Systolic Hypertension in China (Syst-China) Collaborative Group. J Hypertens. 1998;16:1823-1829.

55. Dahlof B, Sever PS, Poulter NR, et al. Prevention of cardiovascular events with an antihypertensive regimen of amlodipine adding perindopril as required versus atenolol adding bendroflumethiazide as required, in the Anglo-Scandinavian Cardiac Outcomes Trial-Blood Pressure Lowering Arm (ASCOT-BPLA): a multicentre randomised controlled trial. Lancet. 2005;366:895-906. 
56. Jamerson K, Weber A, Bakris GL, et al. Benazepril plus amlodipine or hydrochlorothiazide for hypertension in high risk patients. $N$ Engl J Med. 2008;359:2417-2428.

57. Agrawal R, Marx A, Haller H. Efficacy and safety of lercanidipine versus hydrochlorothiazide as add-on to enalapril in diabetic populations with uncontrolled hypertension. J Hypertens. 2006;24:185-192.

58. Data on file. Efficacy and tolerability of a combination of lercanidipine and enalapril in patients with mild to moderate essential hypertension not adequately controlled by enalapril treatment (add-on to enalapril) [CLP1-0019]. Milan, Italy: Recordati Sp; 2004.

59. Data on file. Efficacy and tolerability of a combination of lercanidipine and enalapril in patients with mild to moderate essential hypertension not adequately controlled by lercanidipine treatment (add-on to lercanidipine) [CLP1-0018]. Milan, Italy: Recordati SpA; 2004.

60. Puig JG, Calvo C, Luurila O, et al. Lercanidipine, enalapril and their combination in the treatment of elderly hypertensive patients: placebocontrolled, randomized, crossover study with four ABPM. J Hum Hypertens. 2007;21:917-924.
61. Rump LC. Efficacy and tolerability of the fixed lercanidipine-enalapril combination in the treatment of patients with essential hypertension. Arzneimittelforschung. 2010;60:124-130.

62. Bangalore S, Kamalakkannan G, Parkar S, Messerli FH. Fixed-dose combinations improve medication compliance: a meta-analysis. $\mathrm{Am} \mathrm{J}$ Med. 2007;120:713-719.

63. Waeber B, Burnier M, Brunner HR. Compliance with antihypertensive therapy. Clin Exp Hypertens. 1999;21:973-985.

64. Mancia G, Grassi G, Zanchetti A. New-onset diabetes and antihypertensive drugs. J Hypertens. 2006;24:3-10.

65. Gil Guillén V, Abellan J, Llisterri JL, et al. Efficacy and safety of lercanidipine in combination with enalapril in HBP: preliminary results of ZANYCONTROL study group [Abstract]. Am J Hypertens. 2003; $16: 115 \mathrm{~A}$.
Patient Preference and Adherence

\section{Publish your work in this journal}

Patient Preference and Adherence is an international, peer-reviewed, open access journal focusing on the growing importance of patient preference and adherence throughout the therapeutic continuum. Patient satisfaction, acceptability, quality of life, compliance, persistence and their role in developing new therapeutic modalities and compounds to

\section{Dovepress}

optimize clinical outcomes for existing disease states are major areas of interest. This journal has been accepted for indexing on PubMed Central. The manuscript management system is completely online and includes a very quick and fair peer-review system. Visit http://www.dovepress.com/ testimonials.php to read real quotes from published authors.

Submit your manuscript here: http://www.dovepress.com/patient-preference-and-adherence-journal 